\title{
Towards Learning Strategies on Digital Nomadic Societies
}

\author{
Miguel Gea ${ }^{1}$, Rosana Montes ${ }^{1}$, Miguel Gonzalez Laredo² \\ ${ }^{1}$ Dpt. Lenguajes y Sistemas Informáticos \\ ETSI Informatica y de Telecomunicación \\ ${ }^{2}$ eLearning Centre (http://cevug.ugr.es) \\ University of Granada
}

\begin{abstract}
We are living new paradigms of communication models in modern societies. Digital Age collapsed old-fashioned analogical methods for acquiring knowledge, dissemination and therefore, creating profound changes in our way of life. The Information society is an umbrella term to describe and identify all those changes that are happening in our social structure in the last three decades, and this trend is now focusing towards mobility, flexibility and networking. New teaching methods for new learning skills are required for students. This paper shows the strategic learning trends on Europe towards the consolidation of the Higher Education Area on the adaptation theses new requisites for future nomadic society.
\end{abstract}

\section{Introduction}

Mobility is a key issue in our society. People spend a lot of time working and travelling from one place to another. Technology allows us create new models of communication and collaboration, and that nomadic time is spent in technology-aware places (WIFI, 3G connectivity, fibre channel, Bluetooth) using smart devices (mobile phones, laptops, GPS navigators, etc.). The use of artefacts in everyday life is growing so fast according to the envisioning prediction that the most profound technologies are those that disappear. They weave themselves into the fabric of everyday life until they are indistinguishable from it [1]. This trend of embedding technological advances in society is called Ambient Intelligence, changing the relationships between the computing systems and users [2].

Technology is also related with knowledge sharing and networking in the new space and dimension of global communication. Organisations and information processing is also related with new forms of understanding the work and human relationships. Now the relevance of how other people's activities are part of the context of one's own activities is crucial to understand new form of relationships such as the social networks. This new form of social-aware computing [3] is also present on online communities, that is, groups of people with a common interest or a shared purpose whose interactions are governed by policies in the form of tacit assumptions, rituals, protocols, rules, and laws and who use computer systems to support and mediate social interaction and facilitate a sense of togetherness [4].

These changes also require new models of learning [5] and tools for adapting traditional methods to new envisioning scenarios based on digital citizens. Informal learning [6] is the lifelong process whereby individuals acquire attitudes, values, skills and knowledge from daily activities related to work, family or leisure. It is not organised or structured (in terms of objectives, time or learning support).

On the other hand, we have to adapt formal training to mobile communities. Mobile devices provide a way to intensify this experience generating easy ways of expanding the educational procedures. M-learning [7] is the exploitation of ubiquitous handheld technologies, together with wireless and mobile phone networks, to facilitate, support, enhance and extend the reach of teaching and learning. In [8] is described a system to support the generation of adaptive mobile learning environments. In these environments, students and teachers can accomplish different types of individual and collaborative activities in different contexts.

We have shown some of the current trends affecting any human activity caused by the technological revolution. Section 2 presents some activities that Higher Education in Europe is promoting to adapt and enhance lifelong learning on such direction. Section 3 introduces an example of informal learning based on online communities. Section 4 focuses on virtual mobility as a key issue in formal training and finally, section 5 shows some pending issues and trends. 


\section{The new digital EHEA society}

Europe is involved in a huge change in the Higher Education System in order to create overall convergence at European level [9]. The Executive Agency of the European Commission (EACEA) promotes the Lifelong Learning Programme (LLP), which stimulate learning opportunities across Europe at any stages of their lives. In this frame, the Erasmus Programme (launched in 1987) is one of the best-known Community actions to encourage student mobility and translational cooperation among Higher Education Institutions across Europe.

One of the most profound impacts on nowadays learning, the 'Bologna Process' initiated by the European Commission is aiming at increasing the mobility of European students by removing existing organisational and administrative obstacles. As a benchmark to monitor the progress the European Commission and the Member States agreed that by 2012 a number of at least 3 million individual participants in student mobility should be reached. The 'European Credit Transfer System' (ECTS), the European assimilation process of Higher Education studies (i.e. the Bachelor and Master system) and mobility portals to find available places for studies or internships are undoubtedly important steps to foster the idea of a mobile community of European students. The readiness of students to use the opportunity of Europe-wide studies clearly depends on these organisational and certification issues, but the number of mobile students will also depend on decisions of single students to study abroad, which are strongly influenced by personal, motivational and other self-related considerations.

Mobility of students and teachers has for many centuries been an important aspect of higher education. Today, with the growing importance of internationalisation on the one hand and the increased use of ICT for education on the other hand, both the interest in student/teacher mobility and the possibilities to offer mobility schemes have increased.

Although this is an important issue, the problems arise when students go abroad and find difficulties to integrate in other institutions and countries. Theses experiences constitute an informal learning for these students, and sometimes, this knowledge is lost or only known as own experiences. Thus, when the students prepare their physical mobility, there is an agreement between institutions for ECTS acknowledgments. But sometimes, these changes represent a break-up in their curriculum with few relationships of home and foreign studies. In next sections we propose two approaches to overcome these problems it carry out in physical mobility exchanges.

\section{Online Erasmus community}

In the context of student mobility in the Erasmus Programme, we have developed an online community called Mobi-Blog [10] (the European Weblog Platform for Mobile Students) with the aims at bridging traditional story telling approaches with Web 2.0 applications such as blogs and wikis, at fostering processes of model learning, self refection and independent decision making of European exchange students and at informally supporting learners in formal Higher Education environments.

The goals of Mobi-Blog are the following:

- To provide a multi-institutional, web-based, bottom-up but well-structured and multi-lingual service on European level for peer-to-peer exchange of experiences of individual mobile students containing all not-organisational aspects of mobile studies like motivation, social issues, communication and cultural issues;

- To develop a structured and comprehensive online guide for mobile students;

- To encourage a network of universities in Europe to integrate the Mobi-Blog service into their portfolio of services for mobile students; to gradually complement existing conventional services; and to integrate the Mobi-Blog services to existing virtual campuses;

- To test the innovative learning concept of combining (and linking) the relatively unstructured concept of blogs with the structured offer of an online guide for learning purposes.

A key issue for Mobi-blog is how it can provide a bridge between the 'formal' and 'informal' learning domains. The conceptual framework therefore is incorporating a model of the interaction between learning across different target users, and at different levels of scale, in order to address the project's commitment to developing methods and tools that are applicable at European, national, regional and local levels.

The methodology is largely based on psychological theories of motivation and learning by and through role models [11]. Differing from other theories of learning, the approach of learning with models explains how complex and difficult tasks, for example driving a car, can be learned. For learning with and from a model it is important that the learner feels the model as realistic and as similar to him/herself. During the learning process of the model, some problems should occur before the aim is achieved. The model should be especially interesting and encouraging for the learner. Additionally examples should refer to single persons (not to institutions), which are as similar to the learner as 
possible to enable mechanisms of identification with the model.

Mobi-Blog has also a clear cultural dimension as it provides realistic, first hand information about different cultures and languages in Europe and thereby helps to promote better understanding and tolerance among the different European countries and cultures. For such purpose, we are focusing on a multilingual support to have stories in the native language (using translations mechanism to be available in any language).

In previous research work [12], we analysed different dimensions of such community, such as:

- Population, countries and popular cities where Erasmus students are telling. These data are very helpful to understand the aims and motivations of Erasmus Students telling their stories (nature of destination, cultural differences, etc.)

- Relevant topics of discussion. Although there are several categories and tagging systems, we have grouped the stories on more general areas of interest. These topics represent the most relevant problems and challenges for an Erasmus student on a foreign country (as shown in Figure 1).

- Motivation of choosing the country. It is also important to find out if the selection of the target country is based on a recommendation, a personal decision or a random choice.

- The feeling and affective motivation regarding storytelling. In most of the cases, when people are telling about their day-to-day in a foreign city/country, you can guess (as to the way they tell their story) their positive or negative feelings. Emotional motivation is a key issue in storytelling, and thus in the students' life experience.

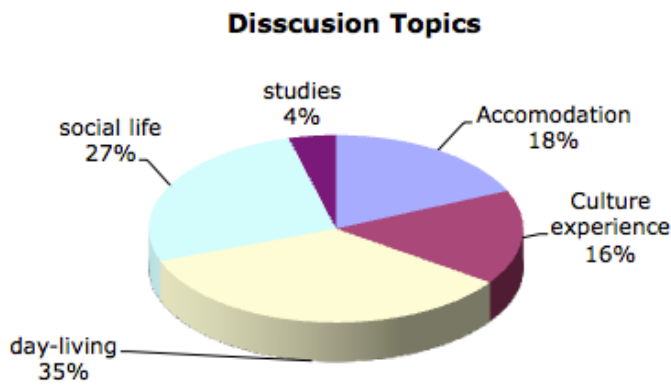

Figure 1. Discussion topics in mobi-blog

For example, the most common topic to talk is about day-living in foreign countries, followed by social life and accommodation. Lesson learnt are really useful for incoming students who have the opportunity to learn form other past experiences. These informal learning are so important because students need to adapt in a completely new environment, and sometimes these information is not institutionally collected or it does not mach with real expectations. Figure 2 shows the student feeling about the story that is telling. The narration denotes that in high percentage, is described positively, so this means a good feedback and credibility for people who is reading.

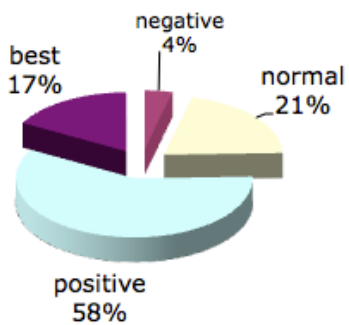

Figure 2. Feeling about mobility experience

Also, the study was supported with a usability analysis (with eye-tracking methods) to understand what user are really interested when they are looking the website. Figure 1 shows the most viewed part of the website, to understand what is relevant for visitors.

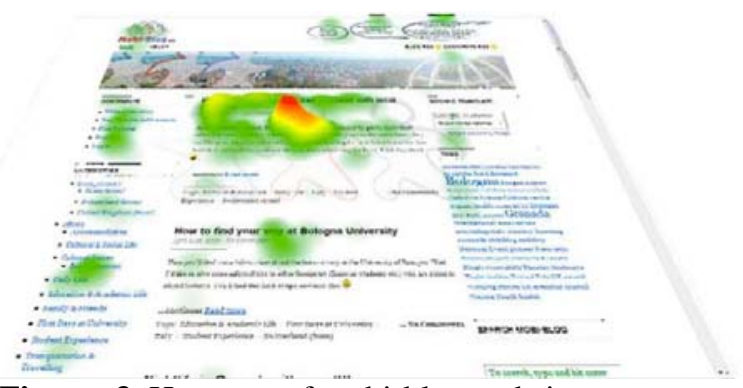

Figure 3. Heatmap of mobi-blog website

This experience shows the need of sharing information on casual online communities, where users only have in common the mobility experience on their studies. This shared knowledge is processed systematically on a online guide to offer institutions a tool to have advises to these frequently asked questions.

\section{Virtual mobility}

Another interesting concept with mobility is the need of persistence in your skill and learning methods. Nowadays, e-portfolios can be considered a novel [13] tool to assess the competences of students independently of their regular classes. Another instrument to achieve that is promoting the virtual mobility as shown in the MOVINTER project [14] we propose to further explore and reformulate the concept of Virtual Mobility in a systematic way. Virtual Mobility is mainly aimed at the 
internationalisation of higher education in a mutual benefit approach, and with respect to the local cultures and the need to valorise existing excellence of education and research in all parts of the world. At first glance [15] Virtual Mobility is defined as the use of information and communication technologies (ICT) to obtain the same benefits as one would have with physical mobility but without the need to travel. But really it's much more than this, virtual courses where part of the activities are done on online communities and combined with face-to-face seminars. Also it may be used as a preparatory activity to physical exchange, enabling a better preparation and follow-up of students who participate in physical exchange programs. So, in order to understand what virtual mobility is, we identify the following relevant features:

- International teaching team, cooperating in the design, implementation, support and evaluation of courses;

- International clusters of students from different countries who mainly study in their local (chosen) university with their fellow students and without going abroad for long periods of time to study; for those students, Virtual Mobility is a way to internationalise

- High interaction and communication through ICT, among the groups of students/teachers based in different countries to discuss diversity depending on national/local/contextual elements;

- Intercultural exchange: the multicultural component constitutes an integral part of the concept of Virtual Mobility. and choice of subjects that justifies the contribution from different countries (especially social sciences, law, economics, but practically any subject in which comparisons from different national contexts may enhance the value of curricula and prepare students for an international social, economical and professional environment;

- Appropriate technological solutions/choices that support the different types of Virtual Mobility;

- Activities that facilitate the communication, the learning and the intercultural exchange. (reflective tools, non-interactive tools, collaborative tools, communication tools, social networking tools)

The other important features that may be present on Virtual Mobility are the following:

- Joint titles, wherever possible, based on a long term confidence relationship

- Among the participating HEIs, built on previous research and teaching cooperation.

- Joint design of curricula, which adds value enormously in terms of reciprocity and mutual benefits between the HEIs in the different countries.

- Joint production of learning resources that enrich the learning experience and the potential of Virtual Mobility.

- The originating vision stresses that the choice of subjects and the design of the learning experience should reflect the advantages of a multi-cultural approach.

These nine elements (shown in figure 2) may change and adapt to the different contexts and models, the expected benefits, outcomes; to the ambitions and needs and the evolving in time.

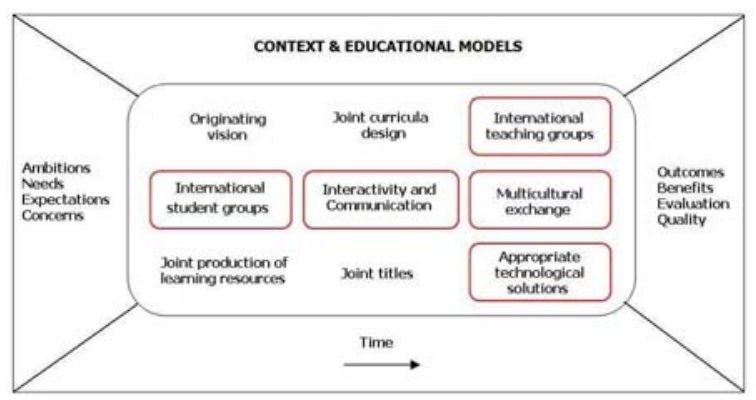

Figure 4. Virtual mobility features

In this context, we can identify three main dimensions in the aim of increasing the potential use of Virtual Mobility. Figure 3 shows the policy, research and community of practice dimensions and related concepts related within.

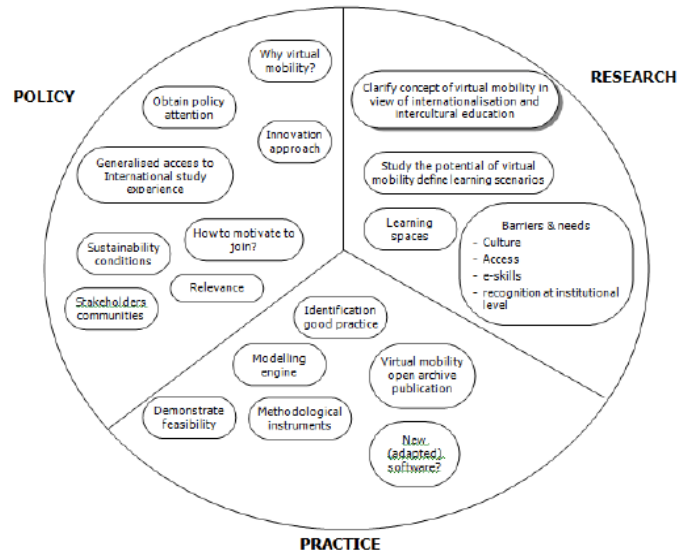

Figure 5. Scopes of the virtual mobility

Virtual mobility can also be achieved to complement physical mobility [16,17], where the technology supports the smooth transition from different models of learning styles. Therefore, virtual mobility is a new emerging model to increase support of nomadic students when (physical) mobility becomes a natural form of specialization and curriculum development. 


\section{The new nomadic society}

Mobility also represents a new model of learning in itself. Learning in context represents an exciting experience where contents are located. Mobile learning (m-learning) allow students to learn anywhere and at any time, enabled by mobile technologies and wireless internet connections, with an interesting feature: the continual changes of location of the learner represent a opportunities to discover services, contents, co-learners or ne different learning contexts [18]. Exploring the potential of those practices and new cultures through the ICT could benefit to teachers and learners by accessing the information just in time and context in their hands. Thereby we orient future work on develop new middleware software which will be able to blend with current platforms for e-Community. The main goal is to create a bridge to easily connect all the key application of the Virtual Mobility. Furthermore, these new challenges are the basis to create community-based system for recommendation or learning taking into account your social awareness [19]. For example, Mobi-blog represents an example of context-aware informal learning from user experience, and this trend should be increased due to their effectiveness for i.e. living abroad by informal learning.

\section{Conclusions and Future research}

In this paper we have introduced the trends towards nomadic society by the emergence of technological revolutions everywhere. These challenges provoke new models of learning we have to achieve: informal learning, context awareness, mlearning, etc. We have analyzed these changes with Erasmus students and we have identified the relevance of enhancing the virtual support to this new models of learning. A further step it the increase of cooperation and internationalization using virtual communities, connected with the creation of student curricula and connected with situated and informal learning. An step toward is the potential support for wider communities adapting contents and context to person with disabilities [20].

Now we have achieved an online community to exchange experiences based on virtual mobility. Future works will be oriented on a systematic approach to improve the opportunities of technology to fully support learning styles based on mobility, collecting interviews of stakeholders and best practices for virtual mobility. Another complementary goal is to propose a service framework to evaluate, design and implement virtual mobility services for internationalisation and academic cooperation.

\section{Acknowledgments and Disclaimer}

This research has been partially supported by the Spanish Ministry of Science and Education, project (MICINN - TIN2010-17344): Adapting Social \& Intelligent Environments to Support people with special needs

\section{References}

[1] Weiser, M.: The computer for the 21st Century. Mobile Computing and Communications Review, Volume 3, Number 3

[2] Aghaja, H., Cózar R.L., Augusto, J.C. (eds.): Human-centric Interfaces for Ambient Intelligence. Academic Press, Elsevier, 2010.

[3] Arroyo, R.F., Gea, M., Garrido, J.L., Haya, P.A., Carro, R.M.: Authoring Social-aware Tasks on Active Spaces, Journal of Universal Computer Science, vol. 14 no. 17: pp. 2758-2776, septiembre de 2008.

[4] Preece, J. (2000) Online Communities: Designing Usability, Supporting Sociability. Chichester, UK: John Wiley \& Sons.

[5] István Bessenyei. Learning and Teaching in the Information Society. eLearning 2.0 and Connectivism. In Information Society, R. Pinter (Ed), Ed. $\quad 2008$. http://www.ittk.hu/netis/doc/ISCB_eng/12_Bessenye i_final.pdf. Access date: 10 May 2010

[6] Kugemann, W.: Learning in the life of digital natives. elearningpapers.eu, vol 14,

[7] Mobile Learning Network (MoLeNET): http://www.molenet.org.uk. Access date: 17 January 2011.

[8] Martín, E., Carro, R.M.: Supporting the Development of Mobile Adaptive Learning Environments: A case study. IEEE Transactions on Learning Technologies, vol. 2, no.1, pp. 23-36, JanMarch 2009, doi: 10.1109/TLT2008.24.

[9] The Bologna Process - Towards the European Higher Education Area.

http://ec.europa.eu/education/higher-education/ doc1290_en.htm. Access date: 10 May 2010

[10] Mobi-Blog: the European Weblog Platform for Mobile Students. http://mobi-blog.eu/, Access date: 17 January 2011

[11] Strahovnik, V., Mecava. B.: Storytelling and Web 2.0 Services: A synthesis of old and new ways 
of learning. eLearning Papers, ISSN: 1887-1542, No 15, June 2009

[12] Gea, M., Arenas, B., Montes-Soldado, R., Luigi Di Stasi, L.: Web 2.0 for Mobile Students: Informal Learning through Storytelling Approaches. EDULEARN'09: the International Conference on Education and New Learning Technologies. Barcelona 2009

[13] Julia Krämer and Günther Seeber: E-portfolios as tools to assess generic competences in distance learning study courses. eLearning Papers, ISSN: 1887-1542, No 6, Sept 2009

[14] MOVINTER: http://www.movinter.eu. Access date: 17 January 2011.

[15] elearning europa.info: definitions: http://www.elearningeuropa.info/main/index.php?pa ge=glossary\&abc=V, Access date: 17 January 2011

[16] VM-BASE: Virtual Mobility Before and After Student Exchanges. http://vm-base.europace.org/

[17] Move-iT: Home \& Away Forum: Coaching Students from a Distance.

http://move-it.europace.org/

[18] Wang, Y., "Context Awareness and Adaptation in Mobile Learning," 2nd IEEE International Workshop on Wireless and Mobile Technologies in Education (WMTE'04), 2004

[19] Farzan, R. and Brusilovsky, P. (2007) Community-based Conference Navigator. In: V. Dimitrova, M. Tzagarakis and J. Vassileva (eds.) Proceedings of 1st Workshop on Adaptation and Personalisation in Social Systems: Groups, Teams, Communities at the 11th International Conference on User Modeling, UM 2007, Corfu, Greece, June 26, 2007

[20] Pérez-Marín, D., Pascual-Nieto, I., Rodríguez, P.: Adapting an educational tool to be used by nonsevere cognitive disabled students. International Workshop Towards User Modeling and Adaptive Systems for All (TUMASA 2009): Modeling and Evaluation of Accessible Intelligent Learning Systems. Brighton, 2009. 